\title{
Solvent Effect on the Enthalpy and Entropy of Activation for the Hydrolysis of Ethyl Cinnamate in Mixed Solvent System
}

\author{
A. K. Singh \\ Department of Chemistry \\ Teerthanker Mahaveer University, Moradabad, India
}

\begin{abstract}
The rate of alkaline hydrolysis of ethyl cinnamate was measured over the temperature range of $20^{\circ} \mathrm{C}$ to $40^{\circ} \mathrm{C}$ in wateracetone mixture at different composition 30 to $70 \%(\mathrm{v} / \mathrm{v})$. The influence of solvent variation on reaction rate was examined in term of changes in the activation parameter. Depletion of $\Delta \mathrm{H}^{*}$ and $\Delta \mathrm{S}^{*}$ value with simultaneous increase in $\Delta \mathrm{G}^{*}$ of the reaction in reaction media, reveals that the reaction is Enthalpy domination and Entropy controlled. The Iso- dielectric activation energy $\left(E_{D}\right)$ of reaction was found to decrease from 52.43 to 47.28 which is calculated by plotting $\log \mathbf{k}$ values against different $D$. The Values of Iso-kinetic Temperature which is less than 300, clearly indicates that there is no appreciable interaction between solvent and solute present in the reaction media, i.e. reaction is not ion-dipole but ion-molecule type.
\end{abstract}

Keywords- Activation Parameter; solvent-solute interaction; Iso-kinetic temperature; specific salvation; Iso Dielectric Activation Energy.

\section{INTRODUCTION}

Solvent play an important role in determining chemical reactivity. In particular, the rate of an elementary chemical reaction may change by orders of magnitude when solvent is changed. Kinetic solvent effect on chemical reaction in different media is correlated in terms of "solvent polarity" which is sums of all the specific and non-specific interaction of the media with initial and transition state". A large effort has been directed towards understanding such solvent effect and a great deal of progress has been made recently [1-3] both theoretically and experimentally. However, the interpretation of kinetic result on the solvent polarity on medium some time fails and sometime succeeds.

In this note we have extended our studies on kinetic solvent on well-known base catalyzed hydrolysis of Ethyl cinnamete which is important for use of adding to cigarettes and cut tobacco that are used for flavor agent and fragrance agent.

\section{EXPERIMENT}

The kinetics of base catalyzed hydrolysis of ethyl cinnamate has been carried out volumetric in water-acetone medium having different concentration of solvent (acetone), varying from 30 to $70 \%(\mathrm{v} / \mathrm{v})$ at five different temperature ranging from $20^{\circ} \mathrm{C}$ to $40^{\circ} \mathrm{C}$ at regular interval of $5^{\circ} \mathrm{C}$. The specific rate constant calculated using second order reaction was found decrease with increase of acetone content, The iso dielectric Activation energy $\left(E_{D}\right)$ is also calculated with help of slope of $\log \mathrm{k}_{\mathrm{D}}$ against $10^{3} / \mathrm{T}(\mathrm{Tab}-\mathrm{I})$ at constant $\mathrm{D}$ and tabulated in Table-II. The iso kinetic temperature was calculated with help of Barclay and Butler relation which come less than 300 .

The evaluated thermodynamic activation parameter has been tabulated in Table-III.

\section{RESULT AND DISCUSSION}

\section{A. Solvent Effect on Rate of Reaction}

In order to discuss the variation of specific rate constant values with gradual addition of dipolar aprotic organic solvent (acetone) in reaction media, the value logk is calculated using second order kinetic. The decrease observed in specific rate constant, with increasing concentration of organic solvent is probably due to depletion of Dielectric constant of media or decreasing polarity of medium as changing from polar water to less polar water-acetone medium. Singh A K [4].

\section{B. Solvent Effect on the Iso-Dielectric Activation Energy of Activation}

From the slope of Arrhenius plot (obtain from interpolation of $\log \mathrm{k}_{\mathrm{D}}$ value against $10^{3} / \mathrm{T}$ at constant $\mathrm{D}(\mathrm{Tab}-\mathrm{I})$ of reaction media. The value of Iso dielectric activation energy of reaction have been evaluated and represented in Tab-II. ( Fig.1 ). It is obvious that $E_{D}$ value goes on decreasing from 52.43 to 47.28 $\mathrm{KJ} /$ mole with increasing in $\mathrm{D}$ value of water -Acetone media, is similar trend to recent finding of Singh R T et al. [5]. 
Table - I

$3+\operatorname{Logk}_{\mathrm{D}}$ Value against $10^{3} / \mathrm{T}$, Water- Acetone System

\begin{tabular}{|c|c|c|c|c|c|c|}
\hline \multirow{2}{*}{ Temp in ${ }^{\circ} \mathrm{C}$} & \multirow{5}{*}{} & \multicolumn{5}{|c|}{$3+\mathrm{Log}_{2}$} \\
\cline { 2 - 7 } & & $\mathrm{D}=60$ & $\mathrm{D}=55$ & $\mathrm{D}=50$ & $\mathrm{D}=45$ & $\mathrm{D}=40$ \\
\cline { 2 - 7 } & $10^{3} / \mathrm{T}$ & $30 \%$ & $40 \%$ & $50 \%$ & $60 \%$ & $70 \%$ \\
\hline $200^{\circ} \mathrm{C}$ & 3.412 & 1.120 & 1.040 & 0.970 & 0.890 & 0.790 \\
\hline $25^{\circ} \mathrm{C}$ & 3.355 & 1.260 & 1.90 & 1.105 & 1.045 & 0.970 \\
\hline $30^{\circ} \mathrm{C}$ & 3.300 & 1.380 & 1.390 & 1.240 & 1.165 & 1.095 \\
\hline $35^{\circ} \mathrm{C}$ & 3.247 & 1.570 & 1.485 & 1.400 & 1.320 & 1.235 \\
\hline $40^{\circ} \mathrm{C}$ & 3.195 & 1.665 & 1.585 & 1.505 & 1.425 & 1.345 \\
\hline
\end{tabular}

Table - II

Values of Iso-Dielectric Activation Energy (water-Acetone media)

\begin{tabular}{|l|l|l|l|l|l|}
\hline $\begin{array}{l}\text { Dielectric } \\
\text { constant(D) }\end{array}$ & $\mathrm{D}=40$ & $\mathrm{D}=45$ & $\mathrm{D}=50$ & $\mathrm{D}=55$ & $\mathrm{D}=60$ \\
\hline $\begin{array}{l}\text { Eexp in } \\
\mathrm{KJ} / \text { mole }\end{array}$ & 52.43 & 51.30 & 50.82 & 48.46 & 47.28 \\
\hline
\end{tabular}

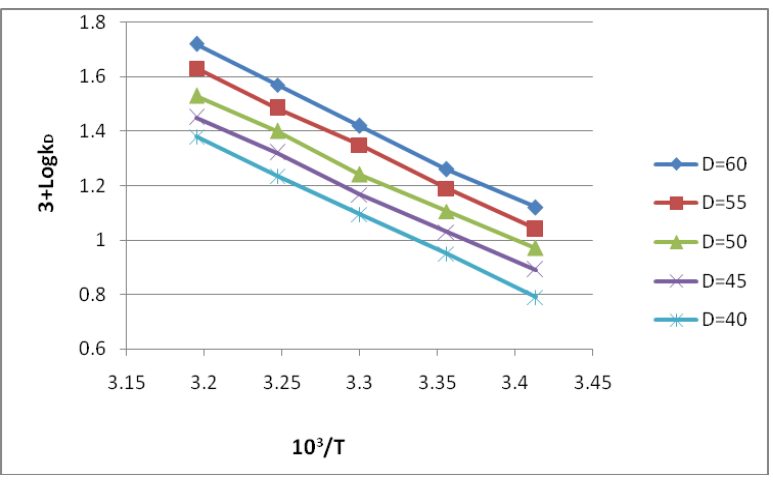

Figure 1. Variation of $3+\log _{\mathrm{D}}$ with $10^{3} / \mathrm{T}$

\section{Solvent Effect and Thermodynamic activation parameters}

The thermodynamics Activation parameter such as Enthalpy of Activation $\left(\Delta \mathrm{H}^{*}\right)$, Entropy of Activation $\left(\Delta \mathrm{S}^{*}\right)$ and free energy of Activation $\left(\Delta \mathrm{G}^{*}\right)$, are better indicator of the solvent effect exerted by the solvent on the solvolysis reaction. These thermodynamics Activation parameter has been calculated with help of Wyne-Jones Eyring[6] equation and absolute rate theory[7] and placed in Tab-III.

On careful observation of the data Tabulated in table III (water-acetone) $\Delta \mathrm{G}^{*}$ value in case of water-acetone increases from 92.09 to $94.26 \mathrm{KJ} / \mathrm{mole}$ with change of proportion of solvents from $20 \%$ to $70 \%$ at $20^{\circ} \mathrm{C}$. Though the enhancement is not large, however it is quite considerable.
The report of Yagil and Anbar[8] indicate that $\mathrm{OH}^{-}$ion of $\mathrm{NaOH}$ is hydrated with three molecules of water. In mixed aquo-organic co-solvent mixture, the organic component molecules are expected to contribute to the process of desolvation as they may be regarded as poor anion solvater in comparison to water. A similar variation in $\Delta \mathrm{G}^{*}$ values with increasing mole $\%$ of organic co solvent has been reported by Tommila [9] and Cleave [10] and recently Singh A K[11]

The variation of $\Delta \mathrm{G}^{*}$ with mole\% in the solvent are nonlinear and increase smoothly with gradual addition of the solvent (Fig. 2 ). This finding is indicative of salvation or desolation of reactant as explained by Absolute Reaction rate theory and supported by Elsemongy [12] and Singh A K. [13] From data mention in the table III, the interesting feature comes in the light is that out of all the value of three thermodynamic parameter $\Delta \mathrm{H}^{*}, \Delta \mathrm{G}^{*}$, and $\Delta \mathrm{S}^{*}$, the value of $\Delta \mathrm{H}^{*}$ and $\Delta \mathrm{S}^{*}$ goes on decreasing with simultaneous increase in $\Delta \mathrm{G}^{*}$ values with increasing proportion of acetone at all the temperature of the reaction, i.e. from $20^{\circ} \mathrm{C}$ to $40^{\circ} \mathrm{C}$. From the fundamental thermodynamic equation

$$
\Delta \mathbf{G}^{*}=\Delta \mathbf{H}^{*}-\mathbf{T} \Delta \mathbf{S}^{*}
$$

it may be inferred that the enhancement in the value of $\Delta \mathrm{G}^{*}$ with simultaneous depletion in $\Delta \mathrm{H}^{*}$ and $\Delta \mathrm{S}^{*}$ value is only possible when the quantitative depletion in the value of $\Delta \mathrm{S}^{*}$ is less than that found in the values of $\Delta \mathrm{H}^{*}$ and from this fact, it is concluded that base catalyzed hydrolysis of Ethyl Cinnamate in water-acetone media is Enthalpy dominating and Entropy controlled. In another word, it may also be inferred that in presence of acetone in the reaction media activate the Enthalpy and control over the Entropy of the reaction.

The change found in the value of three thermodynamic Activation parameter also support the fact that initial state of the reaction is desolvated and the transition state is solvated in the similar ways as recently reported by Singh A K, [14] To explain the solvent effect on the thermodynamic parameters these three values namely $\Delta \mathrm{G}^{*}, \Delta \mathrm{H}^{*}$ and $\Delta \mathrm{S}^{*}$ were plotted against mole\% of acetone in reaction shown in Fig- 2 3and 4 respectively. The nonlinear variation in $\Delta \mathrm{H}^{*}$ and $\Delta \mathrm{S}^{*}$ give information specific salvation taking place in wateracetone as reported by Saviil et al. [15] 
Table-III

Thermodynamics Activation Parameters of the Reaction in Water- Acetone Media $\left(\Delta \mathrm{H}^{*}\right.$ and $\Delta \mathrm{G}^{*}$ in $\mathrm{KJ} /$ Mole, $\Delta \mathrm{S}^{*}$ in $\mathrm{J} / \mathrm{K} /$ Mole)

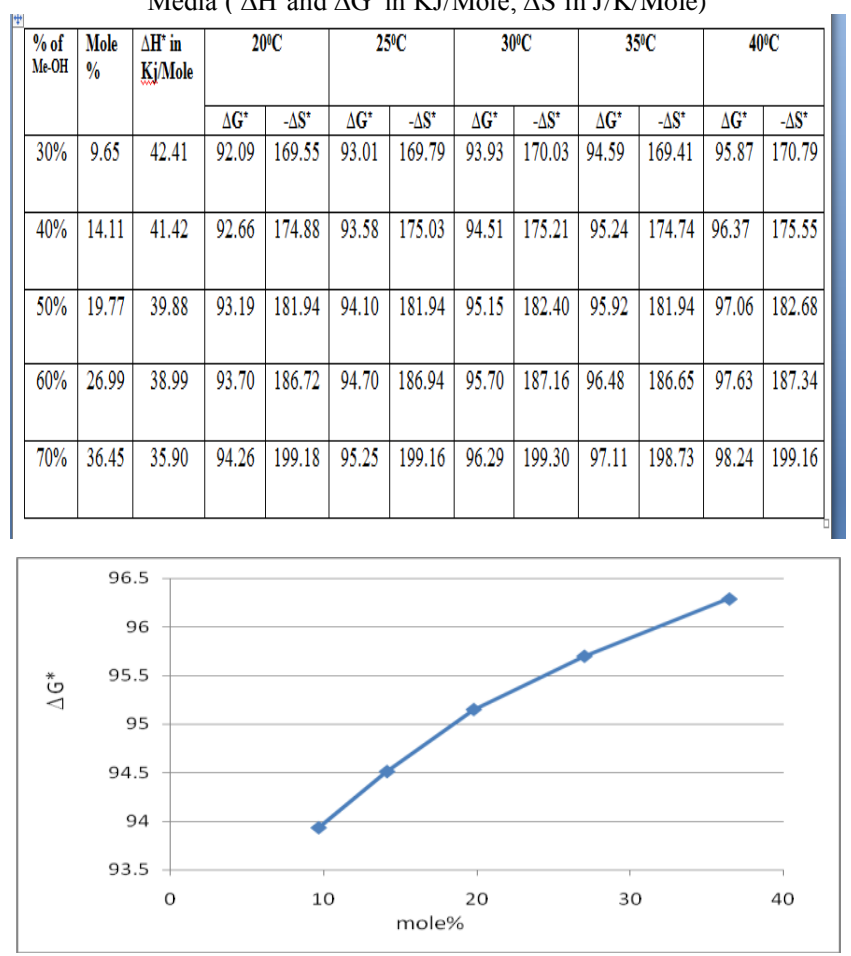

Figure 2. Variation of $\Delta \mathrm{G}^{*}$ with mole $\%$

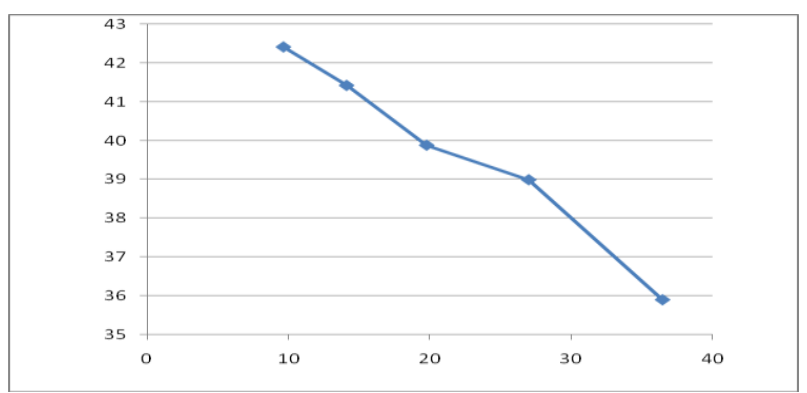

Figure 3. Variation of $\Delta \mathrm{H}^{*}$ with mole $\%$

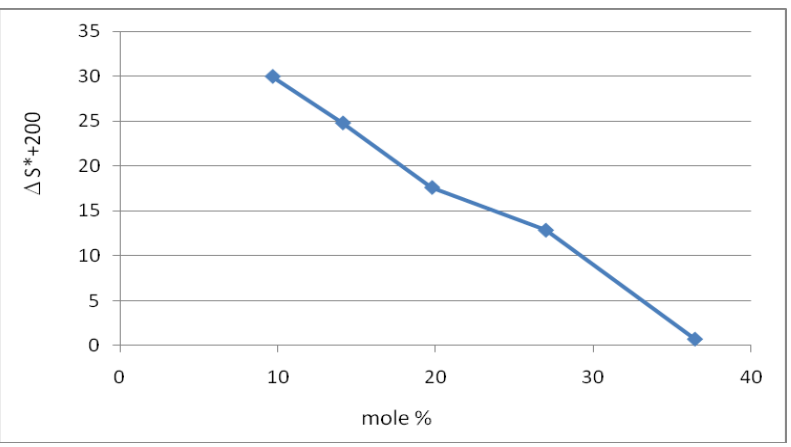

Figure 4. Variation of $\Delta S^{*}+200$ with mole\%

\section{Evaluation of Iso-kinetic Temperature and Solvent-Solute Interaction}

In the light of Barclay and Butler [15] relationship between Enthalpy and Entropy of Activation, which is as follows

$$
\delta \mathrm{m}\left(\Delta \mathrm{H}^{*}\right)=\beta \delta \mathrm{m}\left(\Delta \mathrm{S}^{*}\right)
$$

It is straight line equation representing the relationship between Enthalpy and Entropy of Activation. $\beta$ is the Isokinetic temperature.

From the data available in the table IV the plot of $\Delta \mathrm{H}^{*}$ and $\Delta \mathrm{S}^{*}$ in the solvent system in Fig. 5 and from the slope of straight line, the value of Iso-kinetic temperature was calculated to be 244 (water-acetone) and which is less than 300.

From the value of Iso-kinetic temperature which is much less than 300 , it is concluded that there is slow change in the structure of the reactant or in the solvent or in due to weak interaction between solvent and solute present in the reaction media in similar way as reported by Lefler[16] Our this conclusion has been recently supported by Singh A. K.[18].

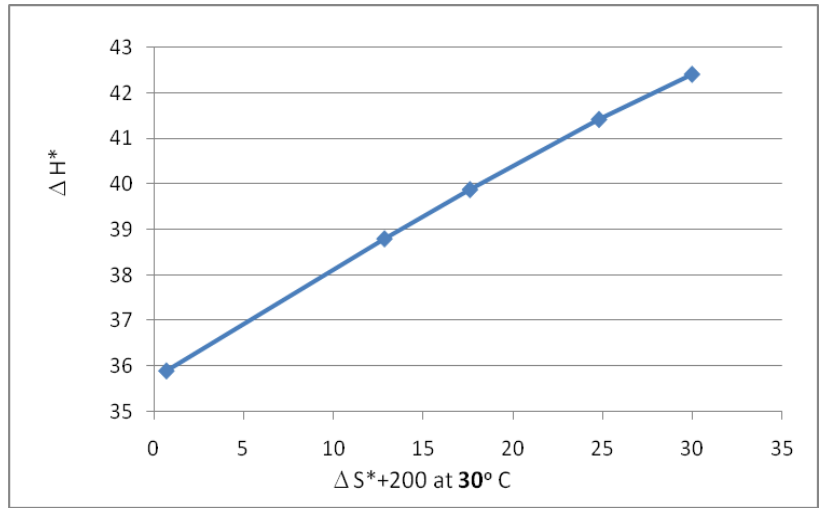

Figure 5. Variation of $\Delta \mathrm{H}^{*}$ with $\Delta \mathrm{S}^{*}+200$ at $30^{\circ} \mathrm{C}$

\section{CONCLUSION}

The result of this work indicate that the rate of hydrolysis of Ethyl cinnamate decreasing trend at all temp witch appear that transition state is more desolveted than initial state. The enhancement in the value of and $\Delta \mathrm{G}^{*}$ with simultaneous depletion in $\Delta \mathrm{H}^{*} \Delta \mathrm{S}^{*}$ for the hydrolysis ethyl inanimate in water- acetone is enthalpy dominating and Enthalpy control. The linear plots obtained by plotting logk as function of $\mathrm{D}$ represent the different electrostatic interaction for the iondipole as well as dipole - dipole reaction. The much less value (>300) of iso kinetic temps indicate weak interaction between solvent and solute.

\section{ACKNOWLEDGMENT}

My special gratitude to my supervisor Prof. R T Singh for his proper guidance and thanks to my friend Dr. Ajit, Dr. Parag and Mr. L K Tiwari for his cooperation during preparation of this content. I am Thankful to Mrs. Rolly Gupta \& Mss. Akansha for their assistance. 


\section{REFERENCES}

[1] Sangita Sarma et al. "Kinetic study of specific base catalyzed hydrolysis of ethyl acrylate in water-ethanol binary system." Russian journal of physical chemistry A, vol-87, issue 5.May 2013 pp730-736

[2] Nada A. Al Jallal. Amer M. Ismail: Solvent effect o kinetics of amide bond cleavage in p. chloro and p. bromo oxazolinanes in Acetone nitrilewater mixture. J. Solution chem., 41, 2154-63, 2012.

[3] Magda F. Fathalla, Kinetics of reaction of 2-chloro-quinosalin with hydroxide ion in $\mathrm{CAN}-\mathrm{H} 2 \mathrm{O}$ and DMSO- $\mathrm{H} 2 \mathrm{O}$ binary solvent mixture" j. Solution Chem., 40, 1258-70, 2011

[4] Singh A K. "The influence of solvent on solvolysis of ethyl cinnamate in water- acetone mixed solvent system." International journal of engineering and applied computer science[IJEACS]. Vol-02, Issue 02, Feb 2017 pp79-82

[5] Singh R T et al. "Kinetic study on the dielectric effect of water-t-butanol media on solvolysis of caproate ester." Napier Indian Advance Research Journal of Science, vol-12 sep 2013, pp78-88

[6] Wynne-Jones W. F. K,and Eyring, H,: “ The Absolute Rate of Reaction in condense Phase". J. chem.. phys. 3, 492-502, 1935.

[7] Glasston, S, Laidler K J and Eyring, H,: The theory of rate process. Mc Graw-Hill Newyork (1941)

[8] Yagil, G. and Anbar N. J., "Classics in solid phase synthesis." J Am. Chem. Soc. 85 (1963) pp 2149-2154.

[9] Tommila. E., Influnce of solvent on reaction velocity. Acta. Chem. Scand 9 (1955) pp957-988.

[10] Cleave, N J ., Soumen Kemi 45B 1935 pp 79-80.

[11] Singh A K. " A kinetic study of solvent effect on thermodynamic activation parameter on alkali catalised solvolysis of methyl salicylate in water-DMF media." International journal of advance Research and innovation. Vol-3, issue-3 2015 pp-547-549

[12] Elsemogy, M.M., Abu Elamayem, M.S., and Mussa, M.N.H.,: Z. Physik chem (Neuetold) 94, 69, 1975.

[13] Singh A K. " Solvolysis rate and activation parameters of ethyl acetate in mixed dipolar organic solvent system-A solvent effect." International journal for research in applied science\& enginnering technology[IJRASET], VOL-4, oct 2016 pp706-710

[14] Singh A K. "A Kinetics Studies of Solvent Effect on Thermodynamic Activation Parameter on Alkali Catalyzed Solvolysis of Methyl Salicylate in Water-DMF Media".(Inter. journal Adv. Reach. And Innovation, volume 3, issue 3(2015)] pp 547-549

[15] Saville B.J. and Hudson, R.E.: J. chem. soc. 4114, 1955.

[16] Barclay L. and Butler, J.A.V: "Entropy of solution.” J. Am. chem. soc. $34,1445,1938$.
[17] Lefler J.E.The Enthalpy- Entropy relation and implication for organic chemistry J. org. chem., 20, 1201, 1955

[18] Singh A. K, "A kinetic study of solvent effect on thermodynamic activation parameter on alkali catalyzed solvolysis of methyl salicylate in water-DMF media". International conferance of advance research and innovation.[ICARI] 2015,pp211-213.

\section{AUTHOR PROFILE}

Dr. A. K. Singh is working as Asst. Professor of Chemistry in College of Engineering of Teethankar Mahaveer University, Moradabad, India since 2010. He holds Master of Science in Physical Chemistry, awarded in 1989, from Magadh University, Gaya followed by Doctor of Philosophy (Ph.D.) in Kinetic Solvent Effect, awarded in 2009 from VKS University Ara, India. He has studied different salvation capacity of

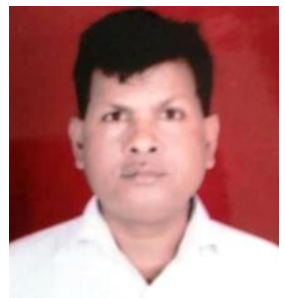
different reaction media (e.g. Water-DMSO, Water-DMF, Water-Aceton. Water-Methanol etc) on the Specific Rate Constant value of the Reaction, the Iso- Composition, Iso- Dielectric Activation Energy, the Number of Water and Solvent Molecules Associated the Activated complex of the Reaction in order to decide the Nature of Mechanistic path of the Reaction, Thermodynamic Activation Parameter of the Reaction, the value of Iso Kinetic Temp on the Reaction in order to Explore the Idea of Solvent- Solute Interaction in More Media. He has published seventeen research articles in his credits. 